\title{
Prophylactic negative pressure wound therapy after lower extremity fracture surgery: a pilot study
}

\author{
Siem A. Dingemans ${ }^{1} \cdot$ Merel F. N. Birnie ${ }^{1} \cdot$ Manouk Backes $^{1} \cdot$ Vincent M. de Jong ${ }^{1} \cdot$ Jan S. Luitse ${ }^{1} \cdot$ J. Carel Goslings ${ }^{1}$. \\ Tim Schepers ${ }^{1}$
}

Received: 18 August 2017 / Accepted: 16 January 2018 / Published online: 29 January 2018

(C) The Author(s) 2018. This article is an open access publication

\begin{abstract}
Purpose Infectious complications following lower extremity fracture surgery are a major concern and account for a substantial socio-economic burden to society. The aim of this pilot study was to investigate the feasibility of a new portable single-use negative pressure wound therapy device in patients undergoing major foot ankle surgery.

Methods Patients undergoing major foot ankle fracture surgery at a single level 1 trauma centre were eligible for this prospective case series. Patient characteristics were collected, as were fracture and surgical characteristics. Primary outcome was surgical site infection within 30 days as classified by the criteria from the Centers for Disease Control and Prevention. Patients in the prospective cohort were case-matched with a historical cohort from the same institution.

Results Sixty patients were included. In seven patients, the NPWT failed and treatment was ceased. Mean age was 44 years and $85 \%$ was ASA $1 ; 43 \%$ of the patients were actively smoking. Indications for surgery were midfoot, calcaneal, talar, and ankle fractures. In 53 patients, four (7.5\%) surgical site infections occurred, two superficial (3.3\%) and two (3.3\%) deep infections. For 47 patients, a match was available. The incidence of surgical site infection did not statistically significantly differ between the prospective cohort and retrospective matched cohort (4.3 versus $14.9 \%, p=0.29$, respectively). This was also the case when looking at superficial and deep surgical site infections separately ( 0 versus $8.5 \%, p=0.08$, and 4.3 versus $6.4 \%$, respectively). Conclusion We have observed surgical site infections in $7.5 \%$ of the patients with the use of prophylactic negative pressure wound therapy. The incidence of surgical site infections was not statistically significantly lower compared to a matched historical cohort.
\end{abstract}

Keywords Negative pressure wound therapy $\cdot$ Postoperative wound infection $\cdot$ Lower extremity fracture surgery $\cdot$ Prophylactic $\cdot$ Closed incision

\section{Introduction}

Infectious complications following orthopaedic (trauma) lower extremity surgery are a major concern. In a prospective study, an incidence of almost $19 \%$ surgical site infections (SSI) following high risk lower extremity fracture surgery was observed [1]. These SSIs are a major burden to the patient and are responsible for a significant increase in (hospitalrelated) costs. Patients who sustain a SSI have prolonged recovery times and are likely to have poorer outcome compared

Tim Schepers

t.schepers@amc.nl

1 Department of Surgery, Academic Medical Center, Trauma Unit, Meibergdreef 9, PO Box 22660, 1100 Amsterdam, DD, Netherlands to patients with an uncomplicated recovery [2], which has been noted in patients following calcaneal and ankle fractures $[3,4]$. In addition, reducing SSIs is not only important because of the negative effect on outcome, but also because of economic costs [5]. A superficial surgical site infection costs an additional $\$ 1000$ extra per patient and a deep infection may cost up to $\$ 20,000$ per case [6-8]. These costs are purely hospital related, and the financial burden to society is likely to be even higher, for example, due to loss of productivity.

The first report of the use of negative pressure wound therapy (NPWT) in orthopaedic surgery dates back to 2006 [9]. The rationale is not yet fully understood but it is believed that negative pressure wound therapy works by (1) increased blood flow through the capillary vessels due to the negative pressure, (2) mechanical stretching of cells leading to cell growth and expansion, (3) compression and reduction of dead 
space, and (4) reduction of oedema and haematoma [1, 10]. Until recently, NPWT was mostly used as a therapy for wound complications (e.g., delayed healing and wound infections) following surgery. Lately, the use of prophylactic NPWT on closed incisions has gained attention. In a randomized controlled trial with prophylactic NPWT in lower leg fractures by Stannard et al., a reduction of almost 50\% (18.9 versus 9.9, $p=0.049$ ) in SSIs was achieved. Furthermore, a lower incidence of wound dehiscence was also noted [1]. These positive results suggest that NPWT may become an adjunct in the attempts to reduce the amount of SSIs in orthopaedic trauma surgery. However, as only one prospective study on prophylactic negative pressure wound therapy in patients undergoing major lower extremity fracture surgery is available, evidence on its beneficial effect is limited and it is uncertain whether the extra expenses of the NPWT device are justified [11]. Additionally, lately portable, single-use devices have been developed, which allow early discharge without the need for specialized home care. It is however unknown whether the good results observed with regular NPWT devices are equaled by these newly developed devices.

The aim of this study was to investigate the feasibility of a new portable single-use negative pressure wound therapy device in patients undergoing major foot ankle surgery. Secondary aim was to compare the incidence of SSI in patients treated with prophylactic NPWT to the incidence in a matched cohort of patients treated with regular dressings.

\section{Methods}

This study was a pilot study to investigate the feasibility of using a new, portable, negative pressure wound therapy device in patients undergoing lower extremity fracture surgery. To allow comparison, the prospective cohort was compared to a historical cohort from the same institution. Patients in this historic cohort had not received prophylactic NPWT. The study was approved by the local Medical Ethical Committee (Academic Medical Center, Amsterdam, Netherlands, 2015_80) and was registered in clinicaltrials.gov registry (NCT02739191). Reporting of the study is in accordance with the Strengthening the Reporting of Observational Studies in Epidemiology (STROBE) guidelines [12]. The study was performed in a single academic centre. Surgeries were performed by experienced, foot/ankle specialized surgeons.

All adult patients scheduled for an orthopaedic (trauma) procedure of the foot and/or ankle (including secondary procedures for treating complications of fracture surgery (e.g., secondary arthrodesis)) with an incision length of at least $3 \mathrm{~cm}$ were considered eligible. The $3-\mathrm{cm}$ threshold for inclusion was chosen to selectively include patients in whom open surgery and not percutaneous surgery was performed.
Exclusion criteria were (1) open fractures or active infections, (2) antibiotic treatment at the time of the operation for a concomitant disease or infection, (3) patients with immune deficiencies, (4) incision location not suitable for negative pressure wound therapy device, (5) inability to adhere to therapy, or (6) incomprehensive understanding of the Dutch language. We hypothesized that a sample of 50 patients would be enough to reliably estimate the incidence of SSI following the use of prophylactic NPWT. With a combined loss to follow-up and failure of therapy rate of $20 \%$, a total of 60 patients need to be included.

The negative pressure wound therapy device used in this study was the PICO ${ }^{\circledR}$ single-use NPWT system (Smith \& Nephew, London, UK). The PICO is a handheld NPWT device without a canister, which maintains a vacuum at \pm $80 \mathrm{mmHg}$. Blood and exudate is absorbed in a nontransparent dressing and partly evaporates through the semipermeable outer layer. The device comes in a package with two dressings and one (battery powered) pump that works for a predetermined duration of seven days.

\section{Treatment of subjects}

Patients were requested to participate in the study prior to surgery and were included after obtaining written informed consent. The NPWT device was applied to the wound directly following surgery at the operation room under sterile conditions. Renasys ${ }^{\circledR}$ (Smith \& Nephew, London, UK) adhesive gel patches were applied around the surgical incision as this is of additive value in maintaining vacuum by the NPWT device. Post-operatively, dressings were changed in case of (1) a saturated dressing, (2) a persistent air leak, or (3) anticipated saturated dressing upon discharge. The NPWT device was active for seven days, after which patients were instructed to remove the dressing and put a regular surgical dressing on the wound. Treatment was considered a failure when (1) treatment duration was shorter than three days or (2) more than three bandages were required to manage wound exudate. Patients were routinely assessed at the outpatient clinic at two to four weeks following discharge. All SSIs occurring during the study period were noted.

\section{Study parameters}

The demographics collected consisted of age, gender, length and weight, American Society of Anesthesiologists (ASA) classification, substance abuse (smoking) and diabetes mellitus. Fracture characteristic were the type of fracture prior to osteosynthesis and in case of a secondary procedure whether a SSI had occurred before. Surgical information comprised types of surgery (i.e., osteosynthesis or arthrodesis), type of incision, duration of surgery, duration of NPWT, and number of bandages used. Device failure was described as one of the 
following: excessive leakage demanding more than three dressing changes, failure of device (inability to remain vacuum), or withdrawn informed consent for reasons related to the device (e.g., device making too much noise).

Primary outcome measure of the study was a SSI as classified by the criteria from the Centers for Disease Control and Prevention (CDC criteria) [13]. Surgical site infections were further subdivided in superficial and deep wound infections. Superficial wound infections were classified as infections treated with oral antibiotics and/or local wound therapy (e.g., debridement at the outpatient clinic). Deep wound infections were described as infections for which intravenous antibiotics and/or a surgical intervention was required. Furthermore, cases with wound dehiscence were noted, which were described as delayed closure of the surgical site without presence of an infection. Patient satisfaction with the NPWT device was measured through a visual analog scale (VAS); zero point represents complete dissatisfaction with the device and ten points represent maximum satisfaction with NPWT device.

\section{Matching}

To allow comparison, patients who received negative pressure wound therapy were case-matched to patients who did not receive negative pressure wound therapy but were treated with conventional surgical dressings. The database with patients available for matching was compiled by performing a retrospective analysis of patients undergoing lower extremity fracture surgery in our center between the first of January 2000 and March 2016. Patients were identified using ICD-9 codes. Characteristics of the patients in the matching database are available in the appendix. Patients who had received NPWT were matched in a 1:1 ratio to patients who had not received NPWT. Matching criteria were type of incision (identical), gender (identical), age ( \pm 10 years), smoking (identical), diabetes (identical), and (in case of secondary procedure) whether they had experienced a SSI following earlier surgery (identical). Matching was performed using R-studio v 3.3.3 (R Foundation for Statistical Computing, Vienna, Austria).

\section{Statistical analysis}

Categorical variables are displayed as counts and frequencies, continuous variables as means and standard deviations or medians, and interquartile ranges where appropriate. Normality was assessed using histograms and plots. McNemars test for related samples was used to compare categorical data, $t$ test for related samples, or Wilcoxon signed rank test for continuous data where appropriate as matched data requires paired testing [14]. A $p$ value $<0.05$ was considered statistically significant. Statistical analysis was performed using SPSS v 23.0 (IBM, Chicago, Ill). A post hoc power analysis was performed using nQuery Advisor v 7.0 (nQuery, Boston, MA).

\section{Results}

The study was performed between April 2016 and February 2017. In total, 88 patients met the inclusion criteria. Twentyeight patients were excluded due to incision location not suitable for negative pressure wound therapy device $(n=6)$, active infection or open fracture $(n=5)$, immune deficiencies $(n=4)$, insufficient comprehension of Dutch language $(n=1)$, inability to adhere to treatment (i.e., mental illness) $(n=5)$, missed inclusion $(n=6)$, or declined participation $(n=1)$ (Fig. 1).

In total, 60 patients gave informed consent and were included in the study. Therapy was not completed in seven patients due to excessive leakage $(n=2)$, inability to maintain a vacuum $(n=4)$, and withdrawn informed consent $(n=1)$. These patients were excluded from the analysis (Table 1). Median duration of therapy in the remaining 53 patients was seven days (IQR 7-7, range 3-7), and 43 (81\%) patients had undergone seven days of therapy. Demographics of the study population and fracture and treatment characteristics are available in, respectively, Tables 2 and 3. More than $82 \%$ of the patients scored therapy with NPWT device with an eight or higher.

\section{Surgical site infections}

A total of four (7.5\%) patients had post-operative wound infections, two superficial and two deep. One superficial wound infection occurred in a 51-year-old smoking female patient who underwent a secondary subtalar arthrodesis through sinus tarsi approach (STA) who had sustained a deep SSI following open reduction internal fixation (ORIF) for a calcaneal fracture three years earlier. She was treated with oral antibiotics and recovered fully (patient 1). The other superficial wound infection occurred in a 75-year-old female with an ankle fracture who was treated with oral antibiotics as well; she also recovered fully (patient 2). One deep infection occurred in a 54-yearold female who underwent primary arthrodesis for a talar fracture through STA. She was treated with intravenous antibiotics and surgical debridement. After the re-operation, she recovered uneventfully (patient 3). The other deep wound infection occurred in a 46-year-old male who presented with local redness at the emergency department three weeks after ORIF of a calcaneal fracture through STA. He was treated with intravenous antibiotics for two and four weeks with oral antibiotics; no surgical debridement was required. He also recovered fully (patient 4). Apart from this, three cases of minor wound dehiscence without presence of an infection were noted.

\section{Matching}

A total of 343 patients were available in the matching database. Details of these patients are available in the appendix. After matching, 47 pairs remained to be evaluated. 
Fig. 1 Flow diagram of the study

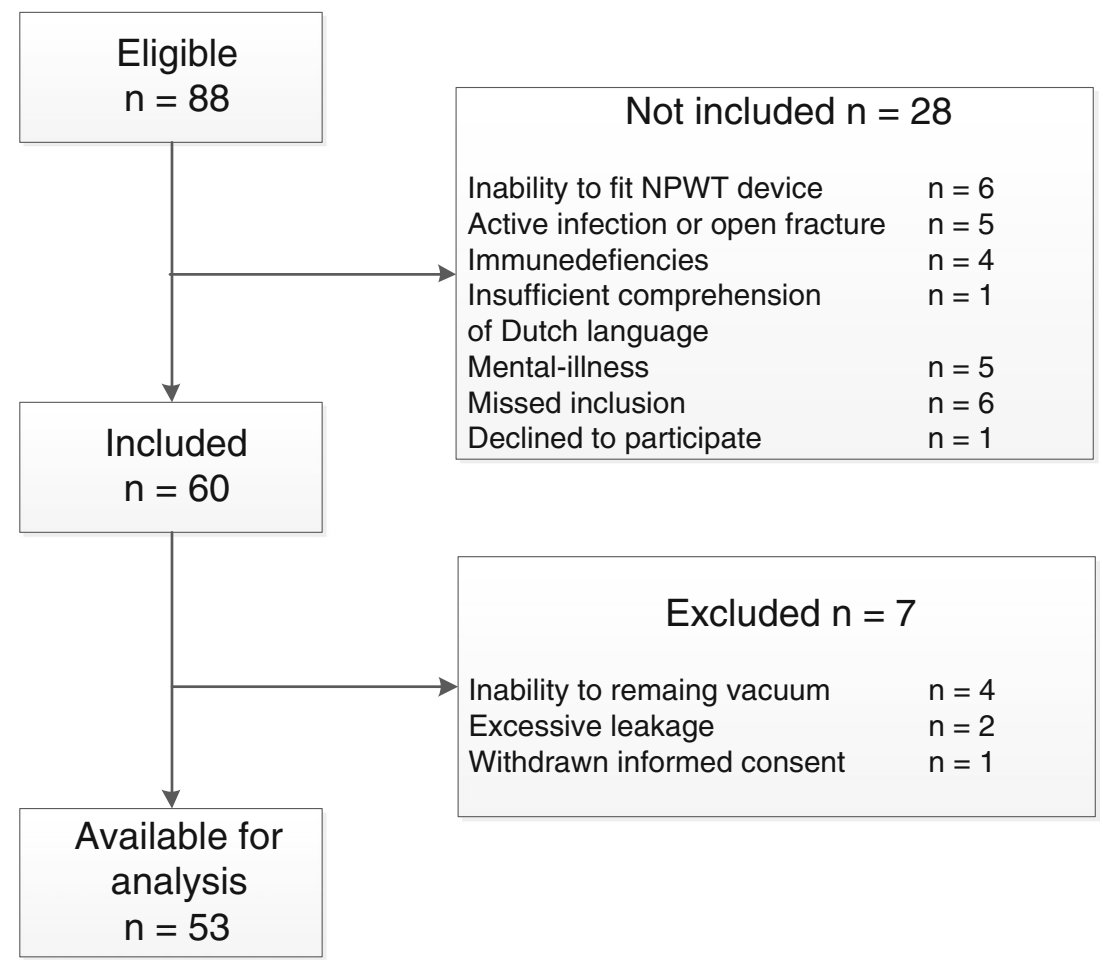

Demographics for the matched patients are available in Table 2. For three patients with a secondary arthrodesis through a STA, no match was found. These three cases had no post-operative wound complications. For one patient undergoing ORIF through STA, no match was found; this patient had no complications on follow-up either. For one patient with a secondary arthrodesis through the extended lateral approach (ELA), no match was found. This patient was patient 1 (described earlier), who sustained a superficial wound infection.
The last patient for whom no match was found was patient 2 , who also sustained a superficial wound infection.

In the 47 matched patients from the historical cohort, seven SSIs had occurred, four superficial, and three deep. The difference in SSI rate between the two groups was not statistically significant (4.3 versus $14.9 \%, p=0.29$, prophylactic NPWT versus conventional surgical dressings, respectively). When looking at superficial and deep SSIs, no statistically significant difference was noted either (superficial 0 versus

Table 1 Excluded patients

\begin{tabular}{|c|c|c|c|c|c|c|c|}
\hline Patient & Gender and age & ASA & Type of surgery & $\begin{array}{l}\text { Earlier } \\
\text { infection }\end{array}$ & $\begin{array}{l}\text { Reason of } \\
\text { cessation therapy }\end{array}$ & $\begin{array}{l}\text { Duration of } \\
\text { therapy (days) }\end{array}$ & Wound complication \\
\hline 1 & Female, 56 years & 1 & $\begin{array}{l}\text { Primary subtalar } \\
\text { arthrodesis through STA }\end{array}$ & NA & $\begin{array}{l}\text { Inability to } \\
\text { remain vacuum }\end{array}$ & 2 & None \\
\hline 2 & Male, 46 years & 1 & $\begin{array}{l}\text { Talar fracture through } \\
\text { medial talar approach }\end{array}$ & NA & $\begin{array}{l}\text { Inability to } \\
\text { remain vacuum }\end{array}$ & 2 & None \\
\hline 3 & Female, 23 years & 1 & $\begin{array}{l}\text { ORIF Lis-Franc through } \\
\text { dorsal foot approach }\end{array}$ & NA & $\begin{array}{l}\text { Inability to } \\
\text { remain vacuum }\end{array}$ & 2 & None \\
\hline 4 & Female, 42 years & 1 & $\begin{array}{l}\text { Secondary subtalar } \\
\text { arthrodesis through ELA }\end{array}$ & Yes & $\begin{array}{l}\text { Excessive leakage } \\
\text { requiring }>3 \text { dressings }\end{array}$ & 1 & $\begin{array}{l}\text { Deep wound infection } \\
\text { for which a re-operation } \\
\text { was required }\end{array}$ \\
\hline 5 & Male, 50 years & 1 & $\begin{array}{l}\text { Secondary subtalar } \\
\text { arthrodesis through STA }\end{array}$ & No & $\begin{array}{l}\text { Excessive leakage } \\
\text { requiring }>3 \text { dressings }\end{array}$ & 2 & None \\
\hline 6 & Female, 70 years & 2 & $\begin{array}{l}\text { Secondary subtalar } \\
\text { arthrodesis through STA }\end{array}$ & No & $\begin{array}{l}\text { Inability to } \\
\text { remain vacuum }\end{array}$ & 2 & None \\
\hline 7 & Male, 50 years & 1 & $\begin{array}{l}\text { ORIF calcaneal fracture } \\
\text { through STA }\end{array}$ & NA & I.C. withdrawn & 1 & None \\
\hline
\end{tabular}

ASA American Society of Anesthesiologists' Score, NA not applicable, STA sinus tarsi approach, ELA extended lateral approach, ORIF open reduction internal fixation 
Table 2 Demographics

\begin{tabular}{lll}
\hline & Patients $(n=53)$ & $\begin{array}{l}\text { Matched cohort } \\
(n=47)\end{array}$ \\
\hline Gender & & \\
Male & $39(74)$ & $36(77)$ \\
Female & $14(26)$ & $11(23)$ \\
Age (mean) [SD] & $43.9[15.6]$ & $42.2[14.6]$ \\
BMI (median) & $24.4[\mathrm{IQR}: 22.2-26.6$, & $24.7[\mathrm{IQR}: 22.4-28.1$, \\
$\quad$ IQR, range] & $18.7-35.5]$ & $16.6-42]$ \\
ASA (\%) & & $31(66)$ \\
1 & $45(85)$ & $13(28)$ \\
2 & $6(11)$ & $3(6)$ \\
3 & $2(4)$ & $0(0)$ \\
Diabetes & & $47(100)$ \\
Yes & $0(0)$ & \\
No & $53(100)$ & $21(45)$ \\
Smoking habits & & $26(55)$ \\
Smoking & $23(43)$ & $0(0)$ \\
Non-smoking & $30(57)$ & $5(100)$ \\
Prior infection ${ }^{\mathrm{a}}$ & & 42 \\
Yes & $1(11)$ & \\
No & $8(89)$ & \\
Not applicable & 44 & \\
\hline
\end{tabular}

ASA American Society of Anesthesiologists' Score, SD standard deviation

${ }^{\text {a }}$ Only patients with secondary arthrodesis

$8.5 \%, p=0.08$, and deep 4.3 versus $6.4 \%, p=0.99$, NPWT versus conventional, respectively) (Table 4).

\section{Post hoc power analysis}

A post hoc power analysis showed that with a significance level of 0.05 and with $80 \%$ power, 236 patients are needed to demonstrate a significant decrease in the incidence of SSI from 14.9 to $4.3 \%$.

\section{Discussion}

In this case series, the single-use NPWT device failed and therapy was ceased in seven out of 60 patients. We observed an incidence of $7.5 \%$ SSI following major lower extremity fracture surgery using prophylactic negative pressure wound therapy. After matching, no statistically significant difference in total number of SSI or in superficial SSI and deep SSI was found between prophylactic NPWT and conventional dressings.

Surgical site infections present a major health care problem, and attempts are made to lower the incidence of infectious complications. Examples are antibiotic prophylaxis [15], repeated administration of prophylaxis [16], less invasive
Table 3 Fracture and treatment characteristics

\begin{tabular}{ll}
\hline & Patients $(n=53)$ \\
\hline Type of fracture & \\
Ankle (\%) & $5(9)$ \\
Talus (\%) & $4(8)$ \\
Calcaneus (\%) & $37(70)$ \\
Mid-foot (\%) & $7(13)$ \\
Time to surgery (days) median [IQR, range $]^{\mathrm{a}}$ & $12.0[10.0-16.0,0-25]$ \\
Type of surgery & \\
ORIF (\%) & $42(79)$ \\
Arthrodesis (\%) & $11(21)$ \\
Incision & \\
Lateral fibula approach (\%) & $5(9)$ \\
Medial talus approach (\%) & $3(6)$ \\
Extended lateral approach (\%) & $4(8)$ \\
Sinus tarsi approach (\%) & $34(64)$ \\
Dorsal foot approach (\%) & $7(13)$ \\
Duration of surgery (min), median [IQR, range] & $89[69-106,35-212]$ \\
Duration of NPWT (days), median [IQR, range] & $7[7-7,3-7]$ \\
Number of bandages used (\%) & \\
1 & $14(26)$ \\
2 & $36(68)$ \\
3 & $3(6)$ \\
\hline
\end{tabular}

$I Q R$ interquartile range, $O R I F$ open reduction internal fixation, $N P W T$ negative pressure wound therapy

${ }^{\text {a }}$ Only ORIF or primary arthrodesis

${ }^{\mathrm{b}}$ Exclusively subtalar arthrodesis

surgical approaches [17], and prophylactic NPWT. A beneficial effect of prophylactic NPWT over conventional dressings was observed in both a meta-analysis of randomized controlled trials and in a meta-analysis of observational studies [18]. One randomized controlled trial in lower extremity orthopaedic trauma surgery showed a significant reduction in the number of SSI from 18.9 to $9.9 \%$ in 249 patients with closed lower extremity fractures [1]. We show similar results with an incidence of SSI of 7.5\%. However, despite a reduction of $7.4 \%$ ( 7.5 versus $14.9 \%$, total SSI incidence in case series versus SSI incidence in matching cohort) to $10.6 \%$ (4.3 versus $14.9 \%$, reduction based on matched cohort), this

Table 4 Matched cohort

\begin{tabular}{lllll}
\hline & \multicolumn{3}{c}{ Matched cohort } & $p$ value \\
\hline & $\begin{array}{c}\text { Cohort } \\
(n=53)\end{array}$ & $\begin{array}{c}\text { Intervention } \\
(n=47)\end{array}$ & $\begin{array}{c}\text { Control } \\
(n=47)\end{array}$ & \\
& $4(7.5)$ & $2(4.3)$ & $7(14.9)$ & $0.29^{\mathrm{a}}$ \\
Infection (\%) & $2(3.8)$ & $0(0)$ & $4(8.5)$ & $0.08^{\mathrm{a}}$ \\
$\begin{array}{l}\text { Superficial } \\
(\%)\end{array}$ & $2(3.8)$ & $2(4.3)$ & $3(6.4)$ & $0.99^{\mathrm{a}}$ \\
Deep (\%) & & &
\end{tabular}

${ }^{\mathrm{a}} \mathrm{McNemars}$ test for related data 
difference was not statistically significant. This was also confirmed by the post hoc power analysis that shows that bigger numbers are needed to achieve a significant difference.

A potential advantage of NPWT is that it requires less changing of dressings compared to regular bandages. Fifty patients in the present study required none or only one change of dressing. This is, according to some, beneficial in the prevention of SSIs as each change of dressings is a potential moment of contamination of the wound. Furthermore, patients were satisfied with the device as they did not have to take care of the surgical wound; they only had to change dressings after one week. In most cases, the wound was dry after one week and dressings were only applied according to the preference of the patient. Furthermore, patients are discharged with the NPWT dressing in situ and do not require specialized home care, which saves costs.

The costs of negative pressure wound therapy have been estimated to be approximately $\$ 500$ per patient [19]. As this does not seem to be a huge expense compared to the costs of a SSI, the evidence for the use of NPWT device need to justify the extra expenses. A recently published paper by Crist et al. showed no beneficial effect of NPWT over regular surgical dressings. Actually, an increase in the incidence of deep SSIs was observed in patients treated with prophylactic NPWT; this was however not statistically significant [19]. This finding underlines the need for thorough additional research on this subject.

An important limitation of this study is that we were unable to find an appropriate match for two patients with a superficial SSI. The first patient (51year-old, actively smoking female who had already experienced a deep SSI following ORIF for a calcaneal fracture) underwent surgery for a secondary arthrodesis through the ELA. From earlier research, we know that patients who have experienced a SSI prior to a secondary procedure are at risk for developing a wound complication [20]. For this reason, patients were matched on this criterion when undergoing secondary procedures. However, as secondary arthrodeses are not performed regularly, we were not able to identify a match for this patient and therefore had to eliminate her from the analysis. For the second patient (75-year-old female, nonsmoking) with an ankle fracture, no match was available either. These types of fractures are generally not treated in our tertiary referral university hospital. As a result, the number of patients available for matching was low. The fact that these two patients were not included in the matching analysis should be noticed, and the results should be interpreted with care. Furthermore, although matching for several factors, which are recognized for being influential on the development of wound complications, it cannot be ruled out that residual confounding exist through factors not matched for. Furthermore, it must be noted that the time span of the historical cohort from which the patients were drawn was
16 years. During these 16 years, major changes in treatment insights may have developed. As a result of this, the SSI rate in the control group may be higher than what could be expected using contemporary techniques; this should be kept in mind when interpreting the results from the matched cohorts.

Lastly, we did not compare wound dehiscence among the two groups. We observed three cases of wound dehiscence in our case series. However, as we collected data on the matching database retrospectively, we felt that we could not reliably identify all cases of wound dehiscence in this group. For this reason, we chose not to compare the incidence of wound dehiscence between the two groups. It is well recognized that the incidence of complications is lower in retrospective studies due to insufficient documentation of complications. Therefore, possible wound complications in patients in the matching database may not have been identified, and the incidence in the control may be higher.

We present the second prospective study on the effect of negative pressure wound therapy in patients undergoing lower extremity surgery and it is the first study using a single-use portable device. Despite a reduction of $>10 \%$ compared to a historical matching cohort, no significant difference was observed. The results of the present study can be used as a benchmark for the development of a future prospective randomized study on NPWT.

\section{Conclusions}

Failure of negative wound pressure therapy was observed in $12 \%$ of patients. We observed surgical site infections in $7.5 \%$ of the patients with the use of prophylactic negative pressure wound therapy. The incidence of surgical site infections was not statistically significantly lower compared to a matched historical cohort who did not receive negative pressure wound therapy. However, the results are promising and a larger study with adequate power could provide more insight in the possible beneficial effect of prophylactic negative pressure wound therapy.

Funding information This study was funded by a grant (IIS 377) from Smith \& Nephew ltd.

\section{Compliance with ethical standards}

Conflict of interest The authors declare that they have no conflicts of interest.

Open Access This article is distributed under the terms of the Creative Commons Attribution 4.0 International License (http:// creativecommons.org/licenses/by/4.0/), which permits unrestricted use, distribution, and reproduction in any medium, provided you give appropriate credit to the original author(s) and the source, provide a link to the Creative Commons license, and indicate if changes were made. 


\section{References}

1. Stannard JP, Volgas DA, McGwin G et al (2012) Incisional negative pressure wound therapy after high-risk lower extremity fractures. J Orthop Trauma 26:37-42. https://doi.org/10.1097/BOT. 0b013e318216b1e5

2. Whitehouse J, Friedman N (2002) The impact of surgical-site infections following orthopedic surgery at a community hospital and a university hospital: adverse quality of life, excess length of stay. Infect Control Hosp Epidemiol 23:183-189

3. Backes M, Schep NWL, Luitse JSK et al (2015) The effect of postoperative wound infections on functional outcome following intra-articular calcaneal fractures. Arch Orthop Trauma Surg 135: 1045-1052. https://doi.org/10.1007/s00402-015-2219-5

4. Schepers T, De Vries MR, Van Lieshout EMM, Van der Elst M (2013) The timing of ankle fracture surgery and the effect on infectious complications; a case series and systematic review of the literature. Int Orthop 37:489-494. https://doi.org/10.1007/s00264012-1753-9

5. Metsemakers WJ, Smeets B, Nijs S, Hoekstra H (2017) Infection after fracture fixation of the tibia: analysis of healthcare utilization and related costs. Injury 48:1204-1210. https://doi.org/10.1016/j. injury.2017.03.030

6. Schrieck-de Loos E, van der Wille J (2009) Sneller beter Doorbraakproject postoperatieve wondinfecties 2004-2008. Best Pract Zorg 242-246

7. de Lissovoy G, Fraeman K, Hutchins V et al (2009) Surgical site infection: incidence and impact on hospital utilization and treatment costs. Am J Infect Control 37:387-397. https://doi.org/10.1016/j. ajic.2008.12.010

8. Berríos-Torres SI, Umscheid CA, Bratzler DW, et al (2017) Centers for Disease Control and Prevention guideline for the prevention of surgical site infection, 2017. JAMA Surg 1-8. doi:https://oi.org/ 10.1001/jamasurg.2017.0904

9. Stannard JP, Robinson JT, Anderson ER et al (2006) Negative pressure wound therapy to treat hematomas and surgical incisions following high-energy trauma. J Trauma 60(6):1301. https://doi.org/ 10.1097/01.ta.0000195996.73186.2e

10. Karlakki S, Brem M, Giannini S et al (2013) Negative pressure wound therapy for management of the surgical incision in orthopaedic surgery: a review of evidence and mechanisms for an emerging indication. Bone Jt Res 22:276-284. https://doi.org/10.1302/ 2046-3758.212.2000190
11. Masters JPM, Nanchahal J, Costa ML (2016) Negative pressure wound therapy and orthopaedic trauma: where are we now? Bone Joint J 98-B:1011-1013. https://doi.org/10.1302/0301-620X. 98B8.BJJ-2016-0373

12. Vandenbroucke JP, von Elm E, Altman DG et al (2014) Strengthening the Reporting of Observational Studies in Epidemiology (STROBE): explanation and elaboration. Int $\mathrm{J}$ Surg 12:1500-1524. https://doi.org/10.1016/j.ijsu.2014.07.014

13. Mangram AJ, Horan TC, Pearson ML et al (1999) Guideline for prevention of surgical site infection, 1999. Hospital Infection Control Practices Advisory Committee. Infect Control Hosp Epidemiol 20:250-278-80. https://doi.org/10.1086/501620

14. Niven D, Berthiaume LR, Fick GH, Laupland KB (2012) Matched case-control studies: a review of reported statistical methodology. Clin Epidemiol 4:99. https://doi.org/10.2147/CLEP.S30816

15. Boxma H, Broekhuizen T, Patka P, Oosting H (1996) Randomised controlled trial of single-dose antibiotic prophylaxis in surgical treatment of closed fractures: the Dutch Trauma Trial. Lancet 347: 1133-1137. https://doi.org/10.1016/S0140-6736(96)90606-6

16. Gans I, Jain A, Sirisreetreerux N et al (2017) Current practice of antibiotic prophylaxis for surgical fixation of closed long bone fractures: a survey of 297 members of the Orthopaedic Trauma Association. Patient Saf Surg 11:2. https://doi.org/10.1186/ s13037-016-0118-5

17. Schepers $\mathrm{T}$ (2011) The sinus tarsi approach in displaced intraarticular calcaneal fractures: a systematic review. Int Orthop 35: 697-703. https://doi.org/10.1007/s00264-011-1223-9

18. De Vries FEE, Wallert ED, Solomkin JS et al (2016) A systematic review and meta-analysis including GRADE qualification of the risk of surgical site infections after prophylactic negative pressure wound therapy compared with conventional dressings in clean and contaminated surgery. Medicine (Baltimore) 95:e4673. https://doi. org/10.1097/MD.0000000000004673

19. Crist BD, Oladeji LO, Khazzam M et al (2017) Role of acute negative pressure wound therapy over primarily closed surgical incisions in acetabular fracture ORIF: a prospective randomized trial. Injury. https://doi.org/10.1016/j.injury.2017.04.055

20. Dingemans SA, Backes M, Goslings JC et al (2016) Predictors of nonunion and infectious complications in patients with posttraumatic subtalar arthrodesis. J Orthop Trauma. https://doi.org/10. 1097/BOT.0000000000000644 